\title{
Radial-velocity and line-profile variations in the sdBV star PG 1325+101*
}

\author{
J. H. Telting ${ }^{1}$ and R. H. Østensen ${ }^{2}$ \\ 1 Nordic Optical Telescope, Apartado 474,38700 Santa Cruz de La Palma, Spain \\ 2 Isaac Newton Group of Telescopes, Apartado 321, 38700 Santa Cruz de La Palma, Spain \\ e-mail: roy@ing.iac.es
}

Received 15 December 2003 / Accepted 13 February 2004

\begin{abstract}
In this paper we present time-series low-resolution spectroscopy of the pulsating sdB star PG 1325+101, obtained with the Nordic Optical Telescope. We have cross-correlated the spectra and Fourier analysed the variations in radial velocity and the width of the cross-correlation function. From both resulting periodograms we easily recover the known main pulsation period. Further analysis of the spectra, after binning them in phase according to the pulsation period, clearly shows evidence for line-profile variations consistent with a radial pulsation mode. The variations show up in all Balmer and several helium lines sampled by the spectra, and can be understood in terms of temperature, gravity and radial-velocity variations. To the best of our knowledge PG 1325+101 is the faintest object in which line-profile variations due to pulsational instability have been detected so far.
\end{abstract}

Key words. stars: subdwarfs - line: profiles - stars: early-type - stars: oscillations - stars: variables: general stars: individual: PG $1325+101$

\section{Introduction}

The hot subdwarf B (sdB) stars are evolved stars commonly found in surveys of faint blue stars (e.g. Green et al. 1986). The general consensus identifies these stars with hot extreme horizontal branch (EHB) stars, implying that they are core helium burning stars enclosed in a thin $\left(M_{\text {env }} \leq 0.02 M_{\odot}\right)$ inert hydrogen dominated envelope (Heber 1986; Saffer et al. 1994). This configuration prevents them from ascending the asymptotic giant branch, and they must evolve instead toward higher temperatures and surface gravities after their core helium is exhausted. Thus, an sdB star turns into a hot sdO star before reaching degeneracy and cooling as a normal white dwarf star (Dorman et al. 1993). However, important questions remain over the exact evolutionary paths and the appropriate timescales.

Stellar seismology is the only way to probe the interiors of stars in detail. When pulsational frequencies and eigenfunctions are known, seismology can put constraints on stellar structure and evolution models. The discovery of pulsations in sdB stars a few years ago, brought with it the opportunity to use asteroseismology to answer the questions about the internal structure and the evolution of these stars.

Kilkenny et al. (1997) were the first to detect photometric variations due to radial and non-radial pulsations in some of the sdB stars. The pulsating sdB stars (sdBVs) have periods

Send offprint requests to: J. H. Telting, e-mail: jht@not.iac.es

* Based on observations from the Nordic Optical Telescope. in the range 100-600 $\mathrm{s}$ with low amplitudes (1-50 millimag). Values of $\log g$ and $T_{\text {eff }}$ for the pulsators are estimated to be in the ranges 5.2-6.1 and 30000-36000 K, respectively. The pulsations in these stars are driven by an opacity bump due to iron and other metallic species (Charpinet et al. 1996) at a temperature of $\sim 200000 \mathrm{~K}$ in the sdB envelope. However, it is a puzzle that some sdBs pulsate while others of identical $\log g$ and $T_{\text {eff }}$ do not. Very recently a group of sdB pulsators with periods on the order of hours and with $T_{\text {eff }}$ in the range $25000-30000 \mathrm{~K}$ has been discovered (also known as "Betsy" stars; Green et al. 2003). Theoretical models have shown that it is possible to explain these pulsations as g-modes driven by the same $\kappa$-mechanism that causes the p-mode oscillations in the short period sdBV stars (Fontaine et al. 2003).

Pulsations produce photometric variations, line centroid variations, line equivalent-width $(E W)$ variations, and moreover line-profile variations (variations in line shape). Whereas photometry, line centroids and $E W$ measurements give information about pulsation frequencies and modal degree $l$, only line-profile variations hold additional information about the azimuthal number $m$ (e.g. Telting \& Schrijvers 1997; Telting 2003). For a full identification of a pulsation mode, the pulsation frequency and the spherical-harmonic quantum numbers $l$ and $m$ of the corresponding eigenfunction have to be known. Once all these modal parameters are known, the observations can confront the stellar structure models to provide constraints on the internal structure of stars. As long as one mode has been 
Table 1. Photometric data on PG $1325+101$.

\begin{tabular}{llll}
\hline \hline$U B V^{a}$ & $V: 13.76$ & $B-V:-0.31$ & $U-B:-1.04$ \\
Strömgren $^{b}$ & $y: 14.019$ & $b-y:-0.134$ & $u-b:-0.267$ \\
$J H K^{c}$ & $J: 14.622$ & $H: 14.764$ & $K_{\mathrm{s}}: 14.866$ \\
\hline
\end{tabular}

Notes: ${ }^{a}$ Green et al. (1986); ${ }^{b}$ Wesemael et al. (1992); ${ }^{c}$ 2MASS all sky catalog.

conclusively identified, and preferably a radial mode, all other observed frequencies can be identified from the modeled frequency spectrum. Without any positive identification, it is difficult to match the frequencies of observed modes with a high degree of certainty, due to the high density of peaks in the calculated frequency spectra.

Jeffery \& Pollacco (2000) were among the first to present a time-resolved radial-velocity study of sdBV stars. Using a drift scan technique to obtain high time-resolution spectroscopy with the $4.2 \mathrm{~m}$ William Herschel Telescope, they detected radial-velocity variations of amplitude $\sim 2 \mathrm{~km} \mathrm{~s}^{-1}$ in the two sdBV stars PB 8783 and KPD 1209+4401. O'Toole et al. (2000) obtained radial-velocity variations of PG 1605+072 using data from three $1.5 \mathrm{~m}$ class telescopes, detecting velocity amplitudes up to $14 \mathrm{~km} \mathrm{~s}^{-1}$ at the same frequencies as the photometric pulsations.

So far only photometric and line centroid/EW measurements of sdB stars have been analysed, but these quantities do not hold enough information to identify a single pulsation mode conclusively. O'Toole et al. (2003) showed that from line-index measurements of PG $1605+072$ additional information on the surface-gravity variations can be derived. We aim to bring the spectroscopic asteroseismological investigations of these stars even further, by doing time-resolved spectroscopy, in order to detect line-profile variations induced by the pulsation modes.

\section{The case for $P G 1325+101$}

The star in this study was found to be a pulsator during a survey using the Nordic Optical Telescope, which identified 10 of the 32 known pulsators of the sdBV class (Silvotti et al. 2000, 2002a; Østensen et al. 2001a,b). The atmospheric parameters of PG $1325+101$ have been derived by others before. Saffer et al. (1994) found $T_{\text {eff }}=34500 \pm 1000 \mathrm{~K}, \log g=6.11 \pm$ $0.15, n(\mathrm{He}) / n(\mathrm{H})=0.021 \pm 0.010$ from least-squares fitting of the Balmer lines with LTE models. Their result confirms the PG classification for this star as an sdB-O star due to the presence of neutral helium. A slightly different result was obtained by O'Donoghue et al. (1998) using a He-free LTE model grid; $T_{\text {eff }}=35900 \pm 250 \mathrm{~K}, \log g=5.90 \pm 0.05$ and $T_{\text {eff }}=$ $34800 \pm 300 \mathrm{~K}, \log g=5.82 \pm 0.07$ from two different observations using different instrumental configurations. The PG catalog (Green et al. 1986) gives a photographic magnitude for PG $1325+102$ of 13.29 (the name PG $1325+101$ occurs first in Saffer et al. 1994), and a photoelectric $B$ magnitude of 13.45 . Photometry collected from the literature and the 2MASS cata$\log$ are compiled in Table 1.

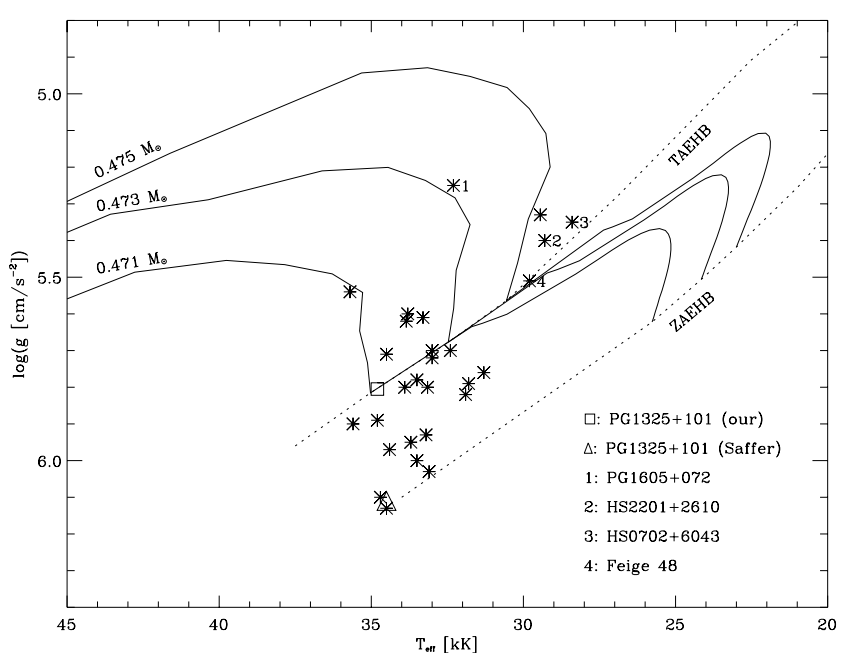

Fig. 1. The positions of a number of $\mathrm{sdB}$ pulsators in the $\left(T_{\mathrm{eff}}, \log g\right)$ diagram, relative to the theoretical zero age and terminal age extreme horizontal branches. Also shown are three evolutionary tracks from Dorman et al. (1993). Note that $0.471 M_{\odot}$ is the lowest mass allowed for an sdB star according to the Dorman models. The fact that most $\mathrm{sdB}$ variables (and most non-pulsators) lie below this track is an unresolved issue.

Saffer et al. (1998) included PG 1325+101 in their radial velocity survey for binarity among degenerate stars. This study found no significant radial-velocity changes, and gives an absolute radial velocity as $+11.5 \mathrm{~km} \mathrm{~s}^{-1}$ with a $1 \sigma$ error of $22.3 \mathrm{~km} \mathrm{~s}^{-1}$.

PG $1325+101$ is one of the most powerful sdBVs in terms of pulsational amplitude of the main frequency at $26 \mathrm{mma}$ (milli modulation amplitudes), only surpassed by PG $1605+072$ at up to $50 \mathrm{mma}$. Unlike PG 1605+072, whose pulsational spectrum is dominated by five periods between $365 \mathrm{~s}$ and $529 \mathrm{~s}$, the spectrum for PG $1325+101$ is dominated by a single stable period at $137.8 \mathrm{~s}$. While PG $1325+101$ is a very typical sdBV star with short period pulsations below about $200 \mathrm{~s}$, PG $1605+072$ is one of only four known sdBVs with stable pulsation periods longer than $350 \mathrm{~s}$ (all marked in Fig. 1). The rarity of the slightly cooler and lower gravity PG 1605+072 type pulsators is consistent with theoretical interpretations that place PG $1605+072$ and its look-alikes at the evolutionary point where core helium is exhausted, and temperature and gravity changes rapidly as it evolves toward the sdO population (Kilkenny 1999). PG $1325+101$, however, may still be core helium burning, sitting right on the extension of the zero age extreme horizontal branch (ZAEHB) if one takes the values for temperature and gravity found by Saffer et al. (1994; see Fig. 1), or closer to or right on the terminal age extreme horizontal branch (TAEHB), if one takes the values from O’Donoghue et al. (1998).

Photometric observations of PG 1325+101 are available from the literature and the 2MASS all sky catalog (see Table 1). The color terms of PG $1325+101$ are typical for an sdB star of around $35000 \mathrm{~K}$, and the infrared magnitudes show no sign of excess that could give away a low-mass main-sequence companion. Although this does not exclude a binary companion, it 
Table 2. Log of spectra used for the frequency analysis.

\begin{tabular}{ccc}
\hline \hline $\begin{array}{c}\text { Date } \\
2003\end{array}$ & $\begin{array}{c}\text { Heliocentric UT } \\
{[\mathrm{s}]}\end{array}$ & $\begin{array}{c}\text { Number of } \\
\text { spectra }\end{array}$ \\
\hline $11 \mathrm{Feb}$. & $11225-26283$ & 401 \\
$19 \mathrm{Feb}$. & $5083-8228$ & 81 \\
$20 \mathrm{Feb}$. & $6752-26742$ & 556 \\
\hline
\end{tabular}

limits the possibilities of a binary companion to either a white dwarf or a low-mass M dwarf.

\section{Observations and reduction}

On the nights of February 10, 18 and 19, 2003 we obtained in total more than a thousand time-resolved low-resolution spectra of PG $1325+101$ with the Nordic Optical Telescope using ALFOSC in long-slit spectroscopic mode (see Table 2). We used a non-standard setup consisting of Grism \#6 and an offcentre slit cut specially for this project, both rotated by 90 degrees to place the spectrum along the CCD serial shift direction. In this way we managed to get the readout time sufficiently short to allow an exposure time of $25 \mathrm{~s}$ and a readout overhead of about $5 \mathrm{~s}$, thus obtaining one spectrum every $30 \mathrm{~s}$. The Loral detector CCD\#7 was binned $2 \times 2$ and windowed to allow 30 arcsec of sky on either side of the object. The off-centre slit allowed us to get a spectral range of about 3200-6100 ̊, with a dispersion of $2.8 \AA$ per binned pixel. The object was acquired onto the slit and monitored until the slit angle rotated too far away from the parallactic angle. Several re-acquisitions onto the slit were done during a night. The total time span of the acquired time series is 9.17 days.

The spectra were reduced using standard IRAF packages. CCD overscan was used to determine the bias level, and bad columns were removed by linear interpolation of pixels in adjacent columns. CCD pixel-to-pixel variations were removed by flat fielding with an internal halogen lamp. One-dimensional spectra were optimally extracted after subtracting a fit to the sky background for each binned detector column. The spectra were normalised first with respect to the mean spectrum of each night, by fitting a cubic spline with 5 to 8 segments to the quotient of each individual spectrum and the mean spectrum. Subsequently, the mean spectrum was normalized to the continuum, using an iterative procedure discarding points lying $2 \sigma$ below a fit of 20 cubic spline segments. The resulting fit was used to rectify the individual spectra. Wavelength calibration was done with helium calibration spectra. The target spectra were clipped to the range of 3650-6000 $\AA$ for our spectral analysis. The peak signal to noise ratio of the reduced spectra was typically around 60. All acquisition times were transformed to the heliocentric frame by correcting for the earth orbit only.

The $25 \mathrm{~s}$ exposure time of the spectra gives rise to phase smearing that reduces the amplitude of the radial-velocity variations, and any other type of variations derived from the spectra. Assuming a sinusoidal variation with the known pulsation period of $137.82 \mathrm{~s}$ the smearing reduces the amplitude by only $5.4 \%$, but the amplitude of a sine with the first harmonic of this period is reduced by $20 \%$.
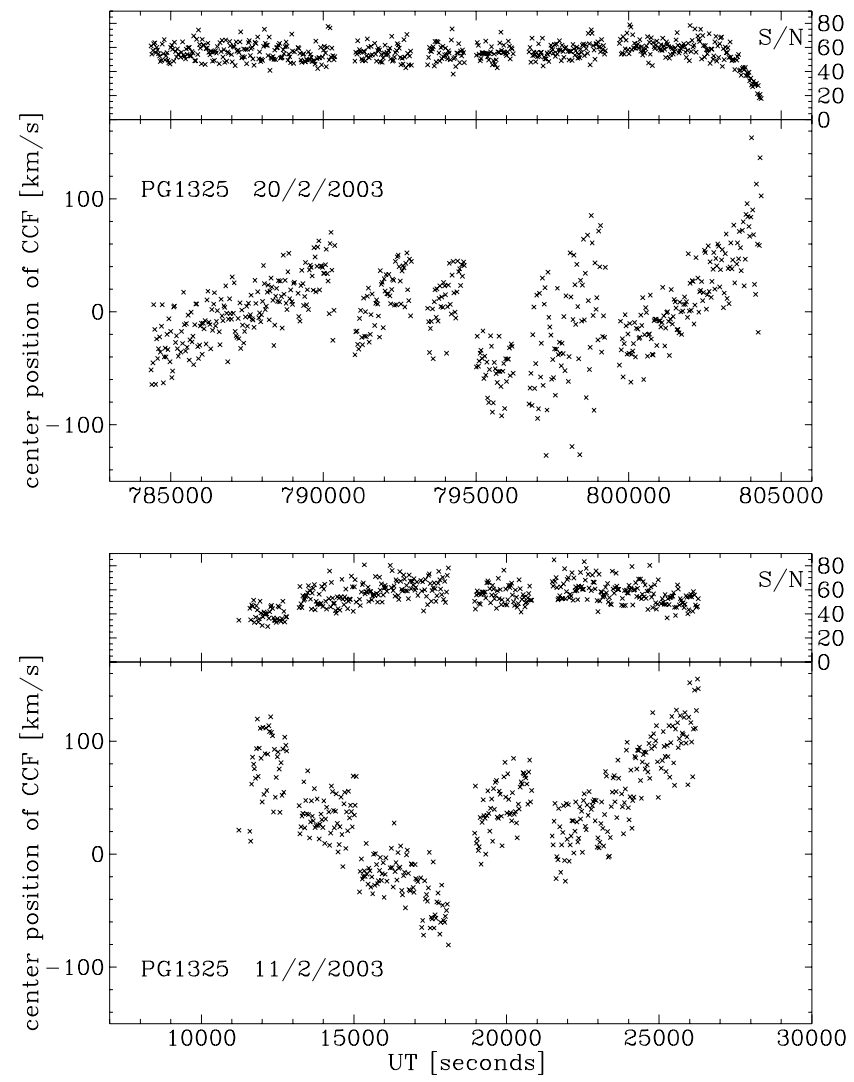

Fig. 2. Center position of the cross-correlation function for two nights of observations, computed with the mean spectrum of each night as template. Signal-to-noise ratio for the individual spectra are indicated in the panels at the top of each diagram.

\subsection{Check for binarity}

The observations described above show radial-velocity variations (see Fig. 2) that could be due to instrumental effects, but that are consistent with a close orbit with a period of 4 to $6 \mathrm{~h}$. In order to check for binarity on such short time scale we reobserved the star on the night of 29 May of 2003, while focusing on obtaining accurate wavelength solutions. We used the ALFOSC Echelle mode using grisms \#13 and \#10, and a 0.5 arcsec slit giving a resolution of $R=3700$ and a wavelength range of 3685-4437 $\AA$. The total time span of the data set of 11 spectra was about $5.5 \mathrm{~h}$, and the exposure times were taken as multiples of the dominant pulsation mode. All spectra were sandwiched by HeNe arc line spectra. These spectra were reduced in a similar fashion as described above. The peak $S / N$ of the extracted spectra ranges between 45 and 85. In Sect. 4.1 we will discuss these spectra with respect to the possibility of a close-orbit binary.

\section{Fourier analysis of radial velocities}

We calculated the radial velocity of the time-resolved spectra using the standard cross-correlation application in IRAF. The mean spectrum of each night served as the template spectrum for that night. The center of the cross-correlation function (CCF) was fit in 12 velocity bins around the maximum with a Gaussian; the 12 bins approximately spanning the points within 


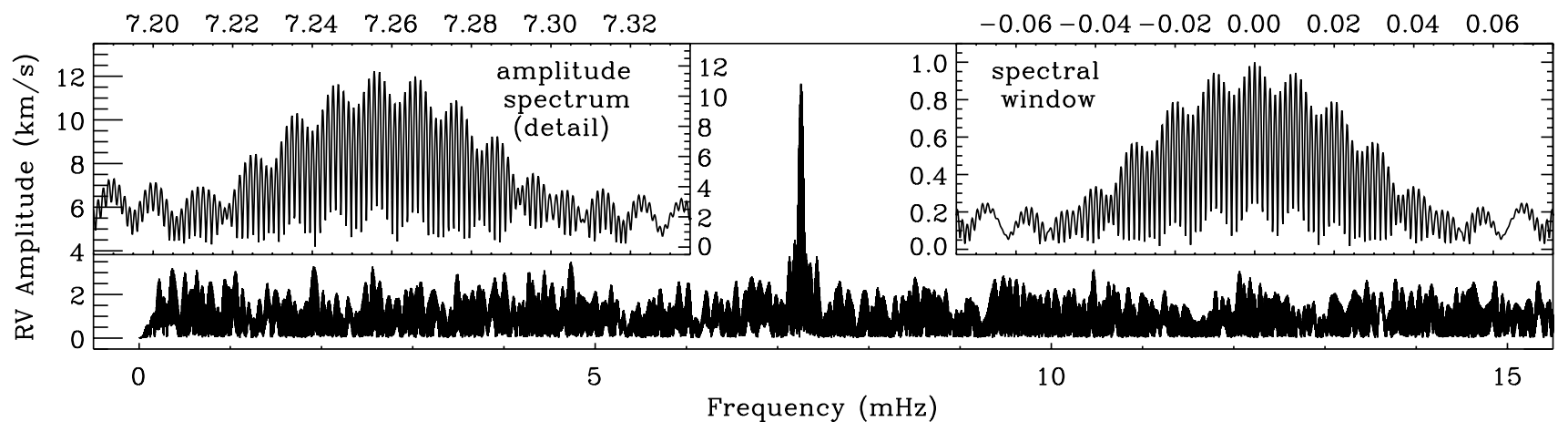

Fig. 3. Fourier periodogram of detrended radial velocities of the best 944 spectra. The blow-up of the main peak shows severe 9-day and 1-day aliasing.

the FWHM of the CCF. We have experimented using different (sections of) template spectra, fitting functions and fit domains, but all gave similar results. The obtained radial velocity shifts are dominated by those of the five strongest lines in the spectrum. The results for two nights are shown in Fig. 2.

From Fig. 2 it is clear that from one telescope pointing to the next jumps in the radial-velocity curve are present, due to the repositioning of the object in the slit. Also between subsequent repointings, there are general trends in the data, which are probably due to flexure in the instrument and drifts in the guiding of the telescope. These instrumental velocity changes appear on timescales much longer than the known pulsation period of the star. Therefore we decided to detrend the observations by fitting straight lines to appropriate chunks of radialvelocity data. The resulting detrended data set has a scatter of $\sigma=19 \mathrm{~km} \mathrm{~s}^{-1}$, which is largely due to atmospheric scintillation on the scale of 0.1 binned pixel ( 0.04 arcsec), which was confirmed by studying the variations in the position of the star in the spatial direction. One stretch of 80 radial-velocity points from spectra taken on the night of 19/2/2003 was discarded because of large scatter possibly due to telescope-guiding problems (see Fig. 2, top diagram).

The Fourier transform of the detrended radial-velocity data is shown in Fig. 3. We note that the detrending only affects the frequencies below $2 \mathrm{mHz}$. The detrended radial-velocity data is shown in Fig. 4, folded with the observed pulsation period. The peak at the pulsation period $P=137.8 \mathrm{~s}$ reflects an observed radial-velocity amplitude of $11.7(0.9) \mathrm{km} \mathrm{s}^{-1}$. This amplitude is subject to the phase smearing due to the exposure time of the spectra, as described in the previous section. Correcting for this effect we find a radial-velocity amplitude of $12.3 \mathrm{~km} \mathrm{~s}^{-1}$. No significant candidate frequencies remain after prewhitening with the pulsation period. The average amplitude of the periodogram after prewhitening with the pulsation period is $1.04 \mathrm{~km} \mathrm{~s}^{-1}$.

The highest peak in the Fourier transform is at frequency $f_{1}=7.25558 \mathrm{mHz}$ (see Fig. 3). The distance between adjacent peaks is $1.3 \mu \mathrm{Hz}$ (9-day aliasing), and the distance between adjacent groups of peaks is $10.3 \mu \mathrm{Hz}$ (1-day aliasing).

A photometric campaign on PG $1325+101$ took place just one month after (between March 21 and Apr. 14, 2003) the spectroscopic data presented here were obtained. The campaign, organised by Roberto Silvotti, involved nine telescopes

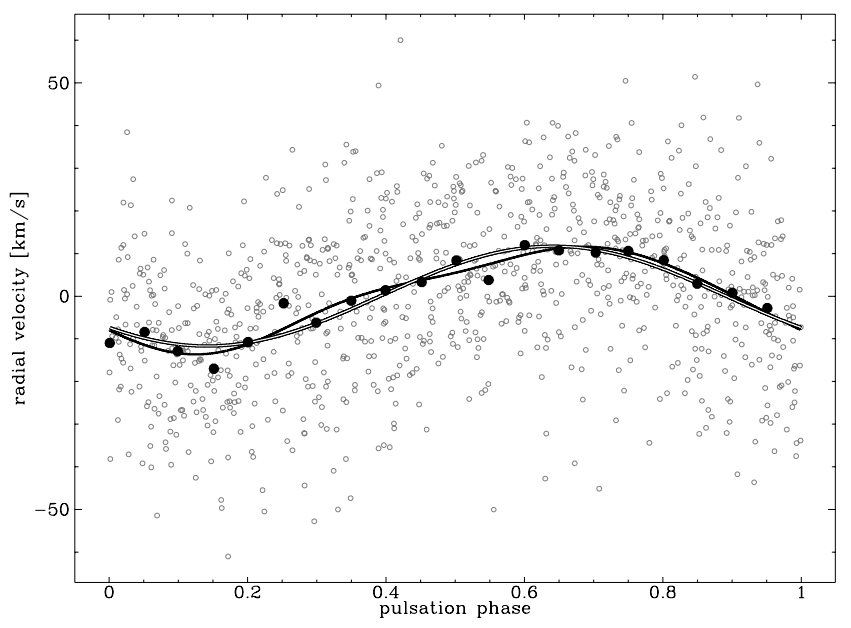

Fig. 4. Radial velocities, obtained from cross-correlation of the timeresolved spectra, subsequently detrended and phased with pulsation period. Also shown are a sine fit, a sine fit with first and second harmonic component and the radial velocities binned in steps of 0.05 in phase.

around the world and obtained more than $250 \mathrm{~h}$ of data (Silvotti et al. 2003). Their preliminary results confirm the persistence of the main $7.256 \mathrm{mHz}$ peak, but they note that the peaks at 7.699 and $9.380 \mathrm{mHz}$, seen in the discovery data from July 2001, have dropped significantly in amplitude (from 5-6 mma to $\sim 1 \mathrm{mma}$ ), well below the detection limit of our spectroscopic data. Thus, the absence of any additional frequencies is consistent with the optical light curve of PG $1325+101$ as observed within a one month interval.

\subsection{Not a close binary}

We used the same cross-correlation technique as described above to analyse the radial-velocity variations of the 11 medium-resolution spectra (see Sect. 3.1), with their mean spectrum as template. We find that the scatter of the individual radial-velocity values around the radial velocity of the mean spectrum is less than $3 \mathrm{~km} \mathrm{~s}^{-1}$. This means that the radialvelocity trends on time scales of one to a few hours as obtained from the low-resolution spectra (see Fig. 2) are due to instrumental effects. 


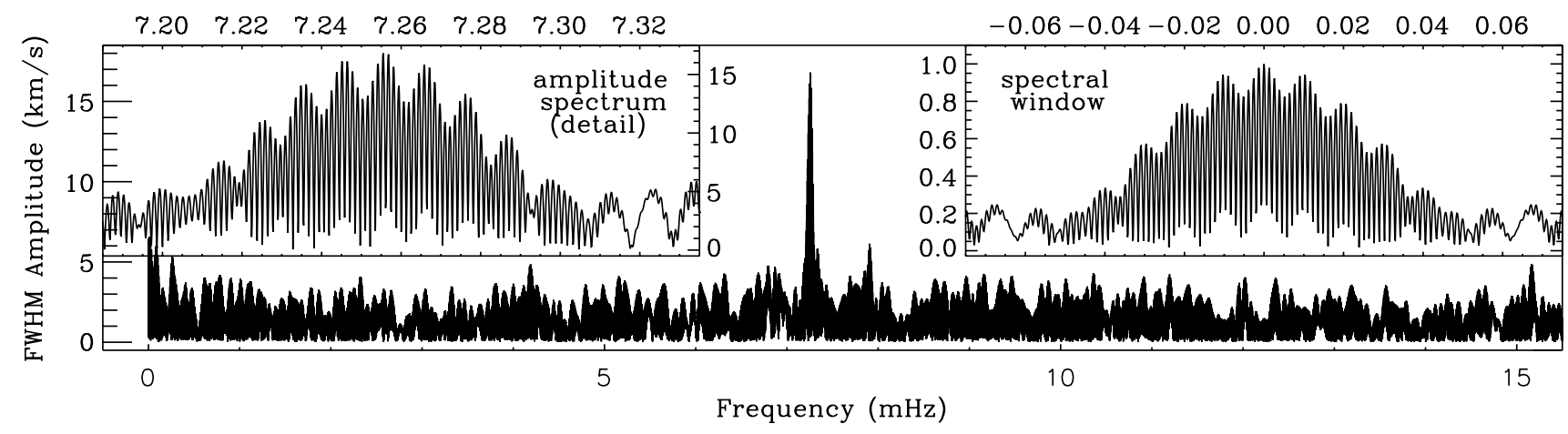

Fig. 5. Fourier periodogram of the $F W H M$ of the CCF for all 1038 spectra.

\section{Fourier analysis of the width of the CCF}

In addition to the position of the center of the cross-correlation function $(\mathrm{CCF})$, taken as the radial velocity, we have also analysed the width of the CCF. Here we used the FWHM of the Gaussian fit to the CCF, and Fourier transformed that width. We used all 1038 spectra in this analysis. No detrending was done.

The peak of the pulsation period is found at $7.25547 \mathrm{mHz}$ in the periodogram (see Fig. 5) with amplitude of $16.8 \mathrm{~km} \mathrm{~s}^{-1}$. The mean amplitude of the periodogram after prewhitening with the pulsation period is $1.48 \mathrm{~km} \mathrm{~s}^{-1}$. Again, no convincing candidates for additional pulsation period were found, although a peak at $7.9042 \mathrm{mHz}\left(5.8 \mathrm{~km} \mathrm{~s}^{-1}\right)$ could be considered if confirmed in other data sets. Since no corresponding peak was found in the radial-velocity periodogram, we do not consider this peak as real.

The clear presence of the main peak indicates that the width of the Balmer lines is changing significantly as a function of pulsation phase (see Fig. 6). As we will discuss in the next sections, this has its origin in the combined effects of surfacegravity and temperature variations.

\section{Line-profile variations in PG 1325+101}

In order to analyse the spectra as a function of pulsation phase, we first shifted all time-resolved spectra to correct for the instrumental radial-velocity drifts (see Sect. 4), and we subsequently folded the spectra in 20 bins according to the pulsation period derived in Sect. 4. Phase zero corresponds to heliocentric UT = 0:00 in the morning of 11 February 2003. The 20 phase-binned spectra have a signal-to-noise ratio in the range $270<S / N<390$.

In Fig. 7 we show the phase-binned spectra and their residuals with respect to the mean spectrum. It becomes clear from the middle and bottom part of Fig. 7 that the behavior of the variations in the lines is similar for all lines that have sufficient signal, except for the He II line at $4686 \AA$ which is in anti-phase with the other lines (the He II line at $5412 \AA$ shows the same behavior but is not included in the plot). This anti-phase behavior is exactly as predicted by the model spectra, since in this temperature range He II lines get more prominent while He I less

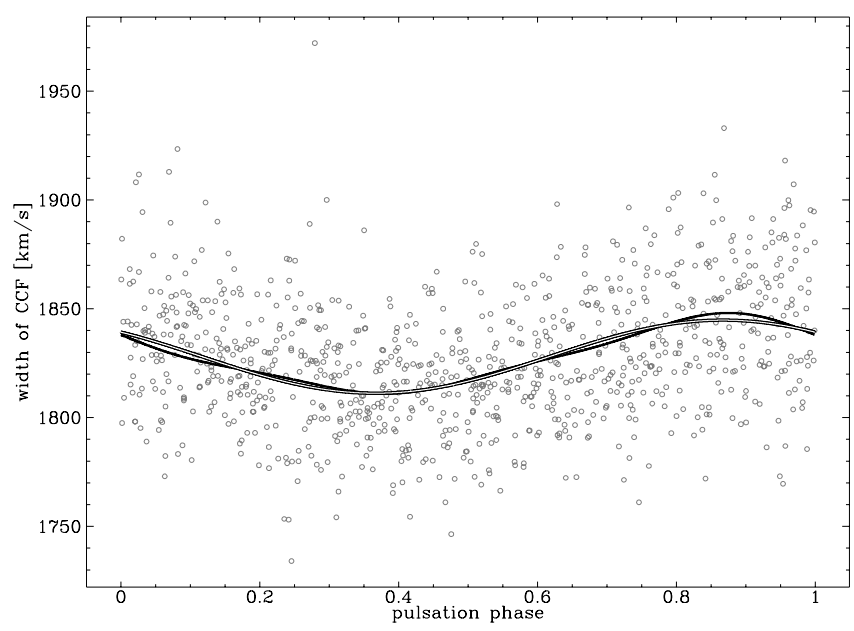

Fig. 6. Width of the CCF phased with pulsation period $\left(f_{1}=\right.$ $7.25558 \mathrm{mHz}$ ). Shown are a sine fit, and a sine fit with first and second harmonic component.

so. It is also clear that the residual variability in the Balmer lines shows up as a from blue to red moving pattern, and that the pattern extends over at least $15-20 \AA$ in the central part of the profile.

As the star shows only one very dominant pulsation mode, we can use the phase-binned spectra to model the modal surface-temperature and gravity changes without too much influence from any other modes in the phased spectra. For this purpose we have used the LTE spectral synthesis model atmospheres of Heber et al. (2000), fitted with the $\chi^{2}$ procedure of Bergeron et al. (1992) and updated to three dimensions by Saffer et al. (1994) in order to incorporate helium abundance into the simultaneous fitting of effective temperature and gravity.

We first used the models to redetermine the continuum in the wavelength region around the high-order Balmer lines of the mean spectrum. Subsequently we adjusted the continuum of the phase-binned spectra according to the continuum correction of the mean spectrum, and refitted the individual phasebinned spectra (see Fig. 8) to yield values of temperature, gravity and helium abundance as a function of pulsation phase. As can be seen from the left panel of Fig. 9, the fitted tem- 


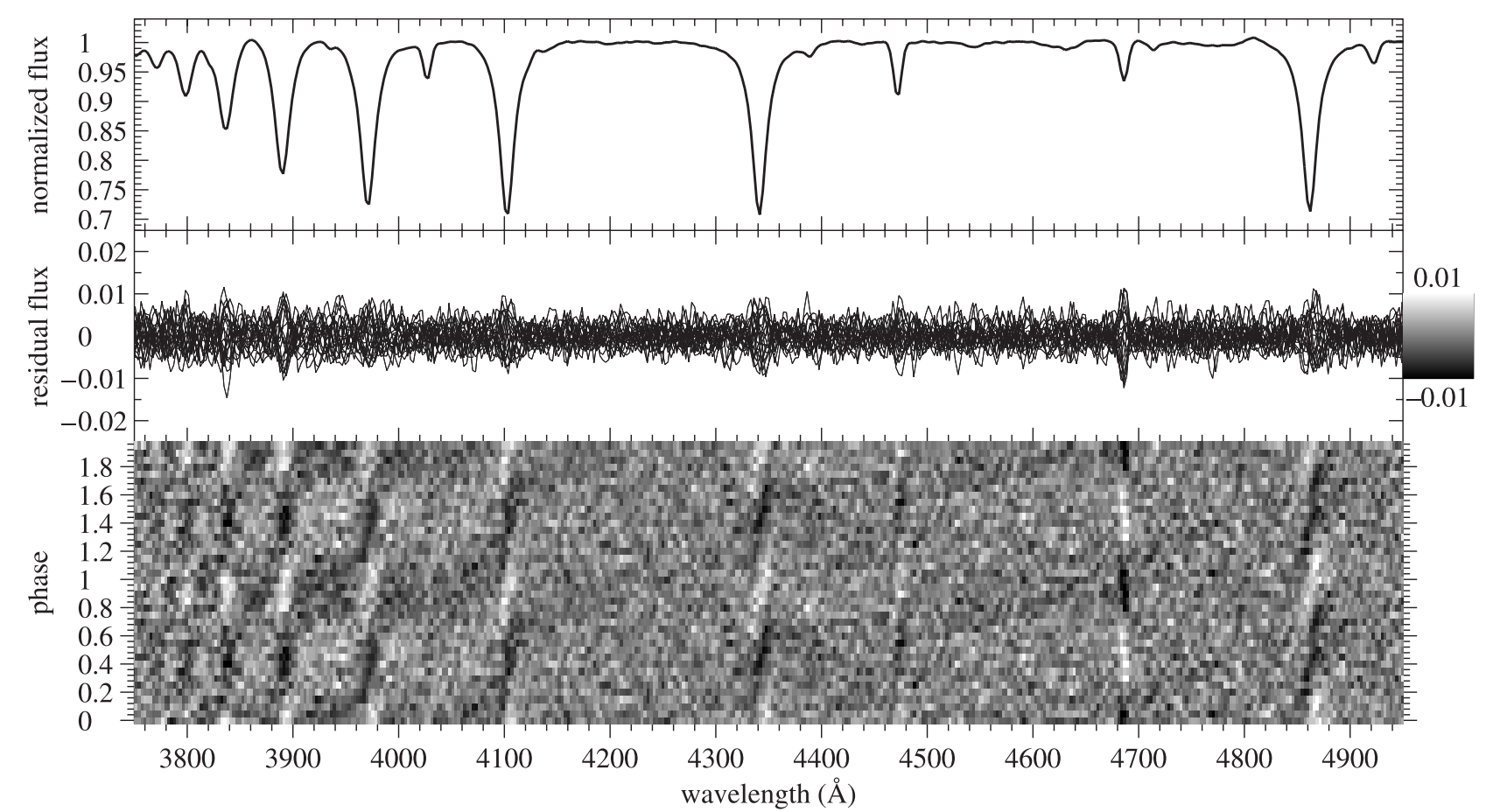

Fig. 7. Mean spectrum and residuals (mean subtracted) of the 20 phase-binned spectra. Two phase cycles of the residuals are plotted in grey scale representation.

perature and gravity varies with the same period as the radial velocity variations, while the helium abundance does not, as one would expect. In the final iteration the helium abundance was kept fixed to the average value found in the previous fit $(\log n(\mathrm{He}) / n(\mathrm{H})=-1.65)$, and the lines were shifted according to the RV found for each phase-binned spectrum. As one can see from the right panel of Fig. 9, this does not change the result much: the formal errors are smaller, and the average temperature differs by only $15 \mathrm{~K}$ and $\log g$ by 0.0015 dex. The mean $T_{\text {eff }}$ and $\log g$ of PG $1325+101$ from the final fit were found to be $34800 \mathrm{~K}$ and $5.805 \mathrm{dex}$, respectively. Systematic changes to these numbers are much more dependent on the choice of lines to include in the fits, than on the other refinements we have made. Figure 8 shows the fits to the lines for the first phase bin when all the 17 lines shown are included (left), when only the Balmer lines are used in the fit (middle), and finally when only the helium lines are used (right). It is clear from the figure that the He II lines in particular point toward a higher temperature than the Balmer lines. The average fit results in the Balmer-line only fits is $T_{\text {eff }}=34660 \mathrm{~K}$ and $\log g=5.798 \mathrm{dex}$, and in the helium-line only fits is $T_{\text {eff }}=37050 \mathrm{~K}$ (with $\log g$ fixed to a value of $5.798 \mathrm{dex}$ ). The formal errors are much larger for the helium-only fits, as expected due to the reduced number of data points in the much narrower lines. The discrepancy between the temperature derived from Balmer lines versus that derived from helium lines has been noted in sdOB stars before (Heber et al. 2000; Edelmann et al. 2003). Although the models have a problem with reconciling the absolute temperatures derived from Balmer and helium lines, the amplitudes of the calculated periodic variation of these values are similar for both cases, and our conclusions regarding the radial velocities are unaffected.
It is clear from Fig. 9 that the variations seen in the profiles can be understood as pulsational surface-gravity and surfacetemperature changes. For a canonical sdB mass of $0.5 M_{\odot}$ the observed mean gravity leads to a radius of $0.15 R_{\odot}$.

\section{Discussion}

As discussed by O'Toole et al. (2003), the radial-velocity variations and surface-gravity variations both hold information on the 3-dimensional pulsational eigenfunction. Here we investigate whether the observed amplitudes of the radial-velocity and surface-gravity variations in PG $1325+101$ are consistent with each other in the case of a radial pulsation mode.

We used the model presented by Schrijvers et al. (1997) to test what amplitude we expect to find for the variations of the center of the CCF of a Balmer line, as a function of the physical amplitude on the star. We used a Lorentzian profile, with depth and width matched to those of the $\mathrm{H} \beta$ line of $\mathrm{PG} 1325+101$, as the intrinsic profile in these calculations. In this exercise we neglected any surface-temperature and surface-gravity effects in the profiles, as these have little influence on the pulsational radial-velocity variations of a radial mode. We find that for a radial mode to result in a CCF radial-velocity amplitude of $12.3 \mathrm{~km} \mathrm{~s}^{-1}$, the physical amplitude on the star has to be $A_{\mathrm{v}}=$ $18.0 \mathrm{~km} \mathrm{~s}^{-1}$. For a sinusoidal pulsation, this implies that the amplitude of the radius variation and the amplitude of the pulsational acceleration are $A_{\mathrm{r}}=A_{\mathrm{v}} \frac{P}{2 \pi}=394 \mathrm{~km}=0.00056 R_{\odot} \sim$ $0.004 R_{*}$ and $A_{\mathrm{a}}=A_{\mathrm{v}} \frac{2 \pi}{P}=0.82 \mathrm{~km} / \mathrm{s}^{2}$ respectively.

From our fits to the model spectra we find that the amplitude of the variation of the measured surface gravity due to the pulsation is $\Delta \log g=0.051(0.005)$ dex (corrected for phase 

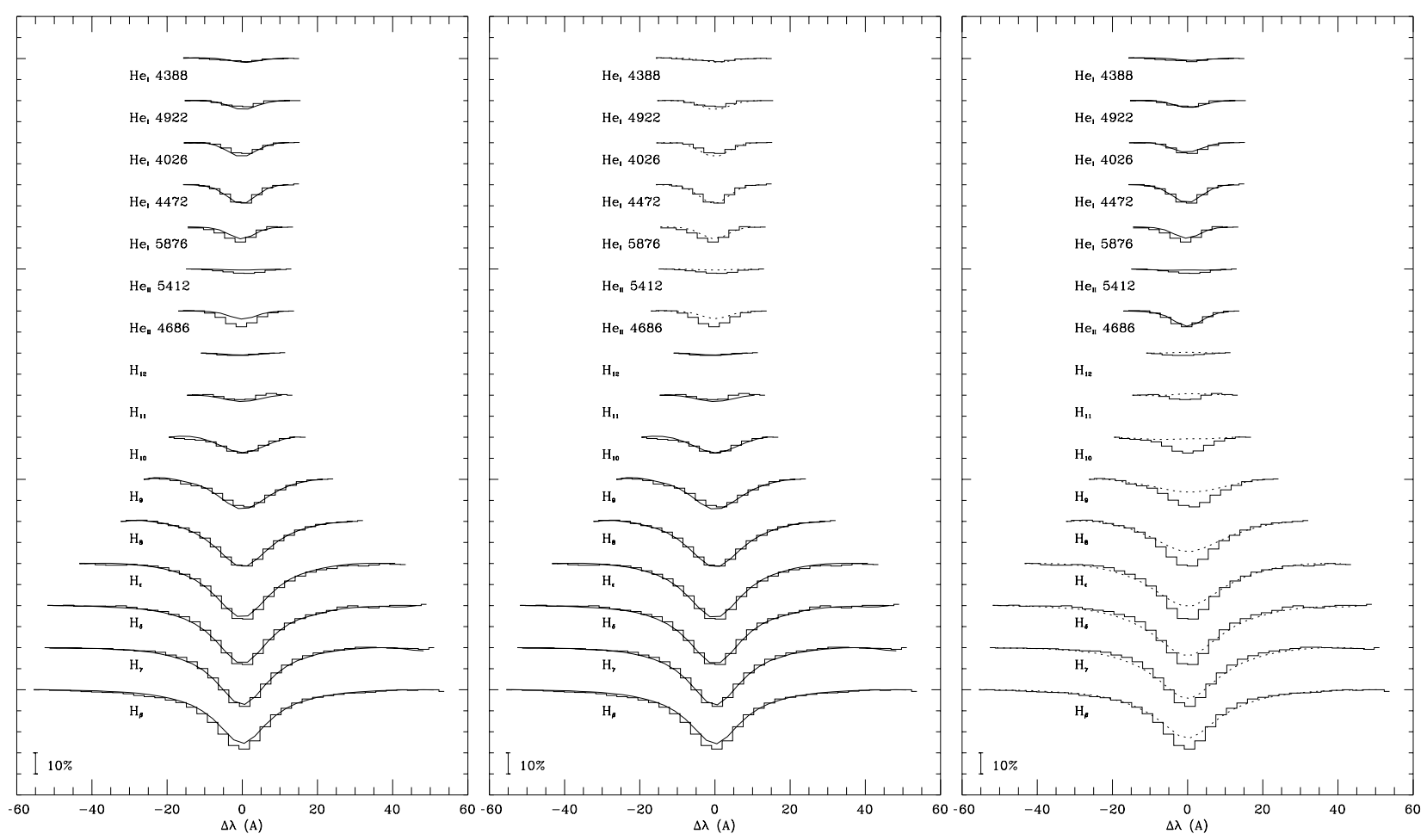

Fig. 8. Model fits to the Balmer and helium line profiles, shown for the first phase bin only. The leftmost plot shows the fit when all lines shown are used in the fitting, the middle one when only the Balmer lines are used, and the rightmost one when only the helium lines are used. The observed data is plotted histogram style, and the model spectra with continuous curves for fitted lines and dotted curves for lines not included in the fit.
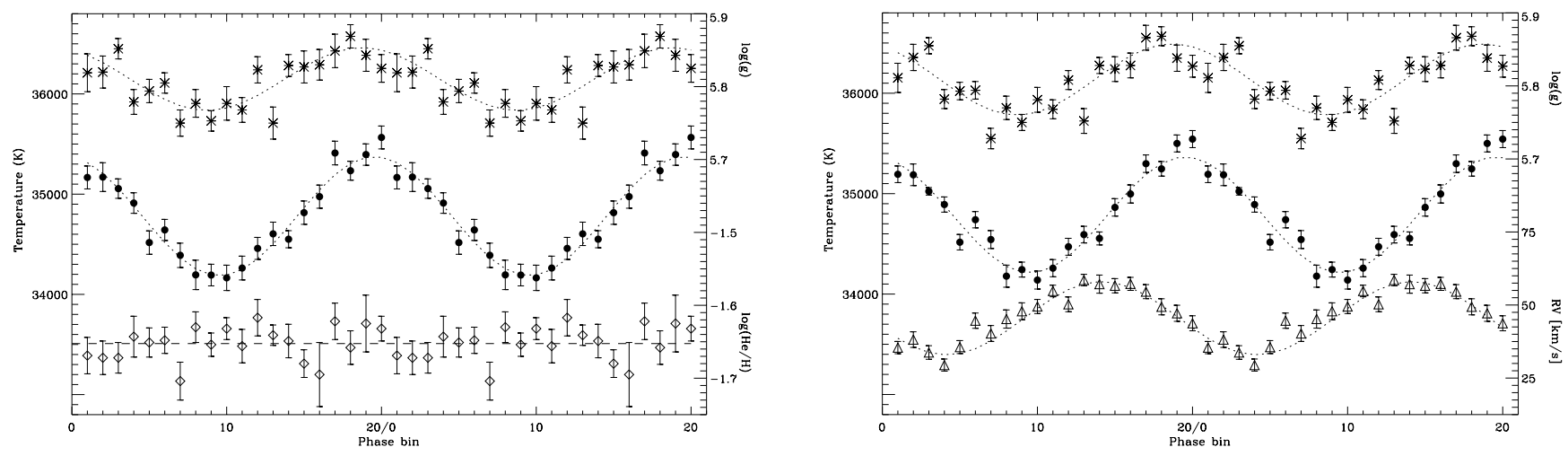

Fig. 9. Results of fitting model spectra as a function of pulsation phase. The left panel shows fit of temperature (bullets), gravity (asterisk) and helium abundance (diamonds) to the spectra when using a fixed radial velocity. Two phase cycles are plotted to guide the eye. The right panel shows the results of the fits to temperature and gravity when using the average helium abundace from the first fit (dashed line in left panel), and radial velocities as fitted to each individual spectrum (triangles in right panel).

smearing). This amplitude was determined by fitting a sinusoid to the variations (see Fig. 9). The amplitude of the radius variation $A_{\mathrm{r}}$ gives rise to $\Delta \log g= \pm 0.003$ dex, and cannot explain the observed surface-gravity variation. On the contrary, the amplitude of the pulsational acceleration $A_{\mathrm{a}}$ gives rise to $\Delta \log g= \pm 0.056$ dex and hence is consistent with the observed gravity variation. Our result is consistent with the findings of De Ridder et al. (2002), who concluded that the effect of the pulsational acceleration on the effective gravity of $\beta$ Cephei stars is an order of magnitude more important than the radius variation.

The phases of the variations in temperature, gravity and radial velocity, were derived from the sine fits (Fig. 9): $\phi_{T}=$ $0.68(0.01), \phi_{\log (g)}=0.64(0.01)$, and $\phi_{V_{\text {rad }}}=0.39(0.01)$ with the phases expressed in $2 \pi$ radians. The phase difference between the gravity and the radial-velocity variations is consistent with the expected value of $\pi / 2$ radians. The phase difference between the gravity and temperature variations is due to the nonadiabatic nature of the pulsation. 


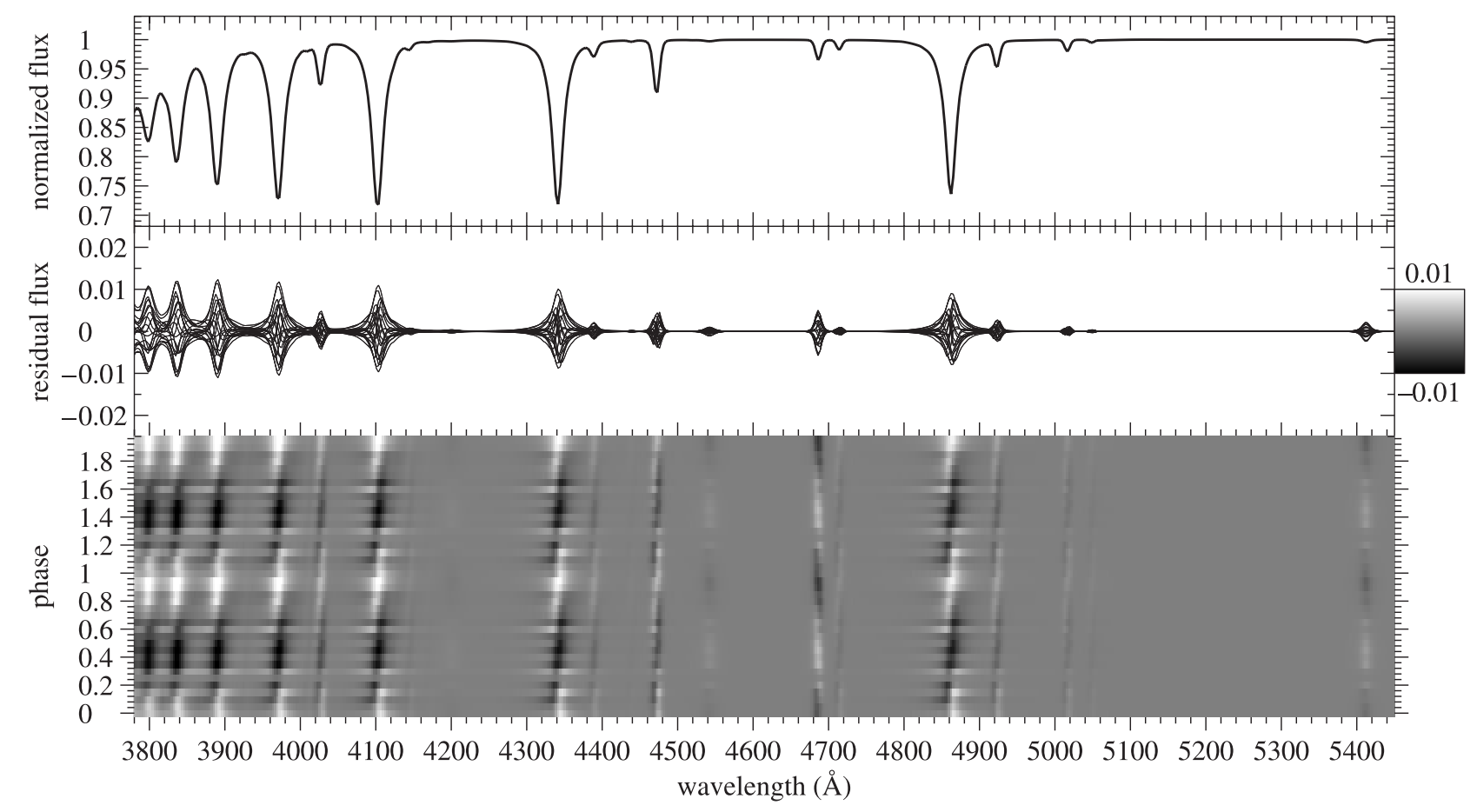

Fig. 10. As Fig. 7 for the model fits to the phase-binned spectra.

It remains to be investigated if the ratio of observed amplitudes of surface gravity and radial velocity depend on the pulsation mode. As shown above, the observed amplitudes in PG 1325+101 are consistent with those expected for a radial mode. Further detailed modeling of these amplitudes, as a function of inclination angle, pulsational degree, and azimuthal order will indicate if the gravity variations hold additional information that can be used to conclusively identify the pulsation mode.

\section{Conclusions}

From a new dataset of more than 1000 time-resolved lowresolution spectra of the pulsating sdB star PG 1325+101 we derived a pulsational radial velocity amplitude of 12.3(0.9) $\mathrm{km} \mathrm{s}^{-1}$, using a spectral cross-correlation analysis. The dominant pulsation frequency is $f_{1}=7.2556 \mathrm{mHz}$. We did not find evidence for other pulsation frequencies in the radial velocity data. When we Fourier analysed the width of the crosscorrelation function, we recovered a clear peak at $f_{1}$, which indicates that the width of the Balmer lines is changing as a function of pulsation phase.

By binning the time-resolved spectra according to the observed pulsation frequency, we have demonstrated clear lineprofile variations in the Balmer and He lines sampled by the spectra. Model fits to the phase-binned spectra showed that the amplitudes of the apparent temperature and surface-gravity variations are 610(30) $\mathrm{K}$ and $0.051(5)$ dex respectively. The average values for PG $1325+101$ are $T_{\text {eff }}=34800 \mathrm{~K}, \log g=$ 5.81 dex, and $\log n(\mathrm{He}) / n(\mathrm{H})=-1.65$.

Our analysis showed that the amplitudes of the radialvelocity and surface-gravity variations are consistent with those expected for a radial mode with $18 \mathrm{~km} \mathrm{~s}^{-1}$ physical amplitude.
However, it remains to be investigated through detailed modeling, whether other modes can lead to similar observed amplitudes, or that the observed characteristics uniquely point to a radial mode.

Acknowledgements. We thank Jacob Clasen for making the custommade slit, Thomas Augusteijn for very useful discussions, and Arto Järvinen for attempting more observations which were not included in this paper because of the mediocre atmospheric conditions under which they were obtained. Furthermore, we thank Prof. Uli Heber for kindly providing the spectral model grids.

The data presented here have been taken using ALFOSC, which is owned by the Instituto de Astrofisica de Andalucia (IAA) and operated at the Nordic Optical Telescope under agreement between IAA and the NBIfAFG of the Astronomical Observatory of Copenhagen. Based on observations made with the Nordic Optical Telescope, operated on the island of La Palma jointly by Denmark, Finland, Iceland, Norway, and Sweden, in the Spanish Observatorio del Roque de los Muchachos of the Instituto de Astrofisica de Canarias.

\section{References}

Bergeron, P., Saffer, R. A., \& Liebert, J. 1992, ApJ, 394, 228

Charpinet, S., Fontaine, G., Brassard, P., \& Dorman, B. 1996, ApJ, 471, L106

O'Donoghue, D., Koen, C., Lynas-Gray, A. E., Kilkenny, D., \& Van Wyk, F. 1998, MNRAS, 296, 306

Dorman, B., O'Connell, R. W., \& Rood, R. T. 1993, ApJ, 419, 596

Edelmann, H., Heber, U., Hagen, H.-J., et al. 2003, A\&A, 400, 939

Fontaine, G., Brassard, P., Charpinet, S., et al. 2003, ApJ, 597, 518

Green, R. F., Schmidt, M., \& Liebert, J. 1986, ApJS, 61, 305

Green, E. M., Fontaine, G., Reed, M. D., et al. 2003, ApJ, 583, L31

Heber, U. 1986, A\&A, 155, 33

Heber, U., Reid, I. N., \& Werner, K. 2000, A\&A, 363, 198

Jeffery, C. S., \& Pollacco, D. 2000, MNRAS, 318, 974 
Kilkenny, D., Koen, C., O’Donoghue, D., \& Stobie, R. S. 1997, Silvotti, R., Solheim, J.-E., Gonzalez-Perez, J. M., et al. 2000, A\&A, MNRAS, 285, 640

Kilkenny, D., Heber, U., \& Drilling, J. S. 1988, SAAOC, 12, 1 359, 1068

Kilkenny, D., Koen, C., O’Donoghue, D., et al. 1999, MNRAS, 303, 525

Østensen, R., Solheim, J.-E., Heber, U., et al. 2001, A\&A, 368, 175

Østensen, R., Heber, U., Silvotti, R., et al. 2001, A\&A, 378, 466

De Ridder, J., Dupret, M.-A., Neuforge, C., \& Aerts, C. 2002, A\&A, 385,572

Saffer, R. A., Bergeron, P., Koester, D., \& Liebert, J. 1994, ApJ, 432, 351

Saffer, R. A., Livio, M., \& Yungelson, L. R. 1998, ApJ, 502, 394

Schrijvers, C., Telting, J. H., Aerts, C., Ruymaekers, E., \& Henrichs, H. F. 1997, A\&AS, 121, 343

Silvotti, R., Østensen, R., Heber, U., et al. 2002, A\&A, 383, 239

Silvotti, R., Bonanno, A., Frasca, A., et al. 2003, Ap\&SS, in press

Telting, J. H., \& Schrijvers, C. 1997, A\&A, 317, 723

Telting, J. H. 2003, Ap\&SS, 284, 85

O'Toole, S. J., Bedding, T. R., Kjeldsen, H., et al. 2000, ApJ, 537, L53

O’Toole, S. J., Jørgensen, M. S., Kjeldsen, H., et al. 2003, MNRAS, 340, 856

Wesemael, F., Fontaine, G., Bergeron, P., Lamontagne, R., \& Green, R. F. 1992, AJ, 104, 203

Zahn, J.-P. 1977, A\&A, 57, 383 